\title{
Compte rendu de Melanesia. Art and Encounter, de Lissant Bolton et al.
}

\section{Gilles Bounoure}

\section{(2) OpenEdition}

Journals

\section{Édition électronique}

URL : http://journals.openedition.org/jso/7168

DOI : 10.4000/jso.7168

ISSN : $1760-7256$

\section{Éditeur}

Société des océanistes

\section{Édition imprimée}

Date de publication : 15 décembre 2014

Pagination : 243-244

ISBN : 978-2-85430-118-2

ISSN : 0300-953x

\section{Référence électronique}

Gilles Bounoure, «Compte rendu de Melanesia. Art and Encounter, de Lissant Bolton et al. », Journal de la Société des Océanistes [En ligne], 138-139 | 2014, mis en ligne le 15 décembre 2014, consulté le 24 septembre 2020. URL : http://journals.openedition.org/jso/7168; DOI : https://doi.org/10.4000/jso 7168

Ce document a été généré automatiquement le 24 septembre 2020.

(c) Tous droits réservés 


\title{
Compte rendu de Melanesia. Art and Encounter, de Lissant Bolton et al.
}

\author{
Gilles Bounoure
}

\section{RÉFÉRENCE}

BOLTON Lissant, Nicholas THOMAs, Elizabeth BONSHEK, Julie ADAMS and Ben BURT (eds), 2013. Melanesia. Art and Encounter, The British Museum Press, 384 p., bibliogr., index, appendice, 306 ill. en couleur, cartes.

1 Ce livre, dont ses responsables et préfaciers revendiquent le caractère hétéroclite comme une incitation et un défi (" a stimulus and a challenge », p. ix), laissera le souvenir d'un pari réussi, même si les 57 contributions qu'il réunit (auxquelles il faut ajouter les textes de présentation des six parties régionales, sud et sud-ouest de la Papouasie Nouvelle-Guinée, nord et Hautes Terres, West Papua, îles Salomon, Vanuatu, NouvelleCalédonie, ainsi que l'introduction de N. Thomas, la conclusion de L. Bolton et l'appendice dû à Geoff Rubenstein) varient considérablement dans leur développement, leur portée et parfois même la nouveauté de leur apport, du moins pour ceux qui suivent attentivement ces domaines de recherche. Il leur semblera d'ailleurs assez clair que ce grand, gros et beau volume est une sorte de petit monument que le British Museum s'est dressé à lui-même par le truchement de sa propre presse et de ses collaborateurs présents et passés. Cette institution, dont on sait combien elle reste fermée aux demandes de restitutions très diverses qui lui sont adressées, entend au contraire se montrer plus ouverte que d'autres aux héritiers ou descendants des communautés dont elle conserve des vestiges, et c'est ainsi qu'a pu y être mené, de 2005 à 2010, sous la direction de L. Bolton, conservatrice du département d'Afrique, d'Océanie et des Amériques de ce musée, le Melanesia Project (Melanesian art: objects, narratives and indigenous owners) dont le présent ouvrage est le résultat le plus spectaculaire et prestigieux. 
2 On n'y lira pas l'histoire de cette vaste et longue entreprise dont subsistent de nombreux documents préparatoires ou rapports d'étape toujours accessibles en ligne, mais seulement des aperçus ponctuels, qui suffisent à prendre la mesure de son ampleur : artistes en résidence à Londres, insulaires du Pacifique invités à visiter les réserves du musée, à y examiner et commenter des objets, enquêtes directes auprès de personnalités mélanésiennes, reconsidération des données muséographiques, etc. Les concepteurs de ce livre, conçu comme "un compagnon » et non un guide "de l'art mélanésien ", ont raison d'affirmer qu'il «aborde ce domaine d'une façon entièrement différente de la plupart de ses prédécesseurs » (p. ix), par la juxtaposition ordonnée d'études de cas, et non sous forme de synthèse illustrée d'exemples représentatifs. De ce point de vue, on pourrait assurer aussi bien qu'il n'a pas de "prédécesseurs ", sauf à se référer à de mémorables catalogues de musées dont les auteurs (par exemple A. Bühler, W. Stöhr, C. Kaufmann, et plus récemment E. Kjellgren pour son excellent Oceania [2007], voir jso 126-127) surent eux aussi prendre les objets comme « compagnons » des connaissances qu'ils dispensaient aux lecteurs.

3 Hormis le thème de la « rencontre " autour des objets d'art, qu'il explore et illustre en tous sens, avec ce leitmotiv sous-jacent que le British Museum est l'endroit où les cultures « se rencontrent » de même qu'elles « dialoguent » au musée du quai Branly, la thèse de ce volume est à chercher davantage dans sa méthode que dans son cheminement et ses conclusions. On pourra s'étonner que ses maîtres d'œuvre, dont certains sont connus pour leur goût de l'hypercritique et de la mise en cause des «dogmes ", ne discutent d'aucune manière ce qu'il faudrait entendre par "Melanesia ", ni les origines contestables du terme, ni ses diverses extensions, se gardant notamment d'évoquer le cas épineux de Fidji. La notion est admise d'emblée dans son acception " usuelle », et c'est également sans autre forme de procès que l'ouvrage suit le parcours classique, d'ouest en est, des arts dits mélanésiens, à l'instar de ses « prédécesseurs ». À la différence d'Art in Oceania (2012, voir jso 136-137) auquel avaient collaboré la plupart des concepteurs du présent volume, Melanesia est exempt de visée polémique et ne prétend pas faire table rase du passé. S'il fallait lui trouver une portée théorique, ce serait dans sa mise en avant de principes très britanniques - au moins dans leur origine -, ceux d'un empirisme spécialement dédaigneux des théories, des catégories et même des frontières entre disciplines et terrains de recherche, avec une diversité des approches en elle-même très instructive.

4 Voilà pourquoi ce recueil - parler d'anthologie ne serait pas hors de propos, notamment pour le choix et la qualité des illustrations - est susceptible d'intéresser des spécialistes de tous horizons, tant les domaines abordés sont nombreux et fréquemment connexes, laissant passer librement de l'ethnographie à l'anthropologie ou à l'histoire de la colonisation, des arts populaires contemporains aux vestiges artistiques les plus anciens, sans querelles de territoires ni lignes de démarcation. Puisqu'il est impossible de présenter ici chacune des 57 contributions, ni même une sélection où tel ou tel chercheur professionnel s'offusquerait sans doute de ne pas se voir cité, on ne retiendra que deux chapitres pouvant servir d'emblèmes à toute l'entreprise (18 et 19, "A Story of Change in Mondika " et "'The Apron is the sister of the bilum' ", pp. 104-111), dus à Peter Solo Kinjap et Paraka, appartenant à la même tribu des Hautes Terres occidentales, et qui témoignent de la vitalité présente de l'art du tressage, à la fois des sacs traditionnels et des robes ou tabliers, à partir des fibres synthétiques tirées des emballages modernes. Ces quelques pages sont à mettre en 
relation avec l'enquête menée récemment par Nicolas Garnier sur ce type de création dans les prisons de Papouasie Nouvelle-Guinée, travail dont les qualités sont signalées dans ce même numéro.

5 On donnerait volontiers pour exemplaires ce volume et l'entreprise qu'il couronne si d'autres grands musées occidentaux étaient susceptibles de s'en inspirer, mais on sait qu'à côté des volontés qui n'y manquent pas, leurs moyens matériels ne cessent de se réduire, y compris pour l'édition des catalogues de leurs meilleures expositions. En publiant sous son seul nom ce fort et beau volume, le British Museum marque là encore la spécificité de son «modèle ", lançant un "défi » qu'il est aujourd'hui le seul en mesure de relever. Dans la situation actuelle, la qualité et l'ampleur des apports de Melanesia font souhaiter que cette institution se mette à nouveau au défi sur d'autres sujets intéressant ses collections océaniennes. 